\title{
GÉNERO Y CAPACIDADES DIRECTIVAS EN COOPERATIVAS DE TRABAJO ASOCIADO. ESTUDIO EXPLORATORIO EN CTAS VALENCIANAS
}

\author{
POR \\ Waldo E. ORELLANA ZAMBRANO* y \\ Amanda CELIS EKSTRAND**
}

\section{RESUMEN}

Son numerosas las investigaciones que desde hace más de dos décadas han investigado posibles influencias de género en el comportamiento en la empresa y en los estilos de dirección. Sin embargo, en el análisis no se han contemplado los aspectos institucionales y organizativos, que juegan un papel relevante en el comportamiento y en el trabajo de los directivos.

Atendiendo a lo anterior, este trabajo muestra las implicaciones organizativas que tiene la forma institucional Cooperativa de trabajo asociado (CTA) y cómo afectan al trabajo directivo. Asimismo, y desde una perspectiva de género, se investigan en CTAs valencianas las capacidades directivas vinculadas a diferencias de género.

Los resultados obtenidos sugieren que la naturaleza del trabajo que realizan las directivas (mujeres) así como su visión del trabajo directivo, responde a un estilo directivo contemporáneo, y tienen un mayor "encaje" con el contexto organizativo de la CTA caracterizado por relaciones entre iguales.

\footnotetext{
* Departamento Dirección de empresas "Juan José Renau Piqueras”. Instituto de Economía Social y Cooperativa (IUDESCOOP). UNIVERSITAT DE VALÉNCIA. Dirección de correo electrónico: waldo.orellana@uv.es

** Becaria de Investigación. Departamento de Personalidad, Evaluación y Tratamientos Psicológicos.

UNIVERSITAT DE VALÉNCIA: Dirección de correo electrónico: ami_cel@yahoo.es
}

REVESCO No 105 - MONOGRÁFICO: La economía social y la igualdad de género -

ISSN: 1885-8031 - www.ucm.es/info/revesco

DOI: 10.5209/rev_REVE.2011.v105.1

Fecha de recepción: 15/11/2010

Fecha de aceptación: 10/01/2011 
Palabras clave: Cooperativas de trabajo asociado, diferencias de género, estilos directivos.

Claves ECONLIT: J160, J540, L200, M190

\title{
GENDER AND MANAGER'S CAPABILITIES IN WORK-COOPERATIVE FIRMS. EXPLORATORY RESEARCH IN COOPS IN VALENCIA (SPAIN)
}

\begin{abstract}
In the last two decades, much research has been done regarding gender differences and leadership styles. This paper presents some of the organizational implications of cooperative firms (Coops) and how their characteristics affect the leadership style of managers. Also, from a gender perspective, we study the management style associated with gender differences in Coops in Valencia (Spain).
\end{abstract}

The results suggest that the nature of the work done by women managers, and their vision of management, responds to a more contemporary leadership style, and fits better in the organizational context of coops, which are characterized by equal relations.

Keywords: Work cooperative organizations, gender differences, management styles.

\section{INTRODUCCIÓN}

El estilo directivo, o la forma de ejercer el liderazgo, es un tema que ha sido ampliamente tratado en la literatura organizativa sin que se haya llegado a un consenso sobre qué estilos son los más eficientes para conseguir que las personas aporten su talento y su potencial, a la obtención de los objetivos empresariales.

Algo similar sucede con las diferencias en los estilos de liderazgo atribuidas a diferencias de género: no existe consenso en las investigaciones, dándose el caso que trabajos como los de Vecchio (2003) y Eagly y Carli (2003), que son de los más citados en la literatura especializada, obtienen resultados opuestos.

REVESCO N 105 - MONOGRÁFICO: La economía social y la igualdad de género -

ISSN: 1885-8031 - www.ucm.es/info/revesco 
En efecto, Vecchio (2003) a partir de una revisión de los resultados obtenidos en investigaciones sobre liderazgo y género, concluye que no existen evidencias que indiquen diferencias significativas en los estilos de liderazgo de hombres y mujeres, ni de que se haya podido probar la existencia de una clara superioridad por parte de uno de los sexos.

Una posición similar a la anterior adoptan Stewart y McDermott (2004), al argumentar que las diferencias encontradas en los estilos de dirección atribuibles a diferencias de género, se deben a que las investigaciones suelen ignorar la variabilidad existente dentro de cada género, lo que induciría a encontrar diferencias. Sostienen, asimismo, que en mujeres y hombres cabe encontrar cualidades atribuidas a uno solo de los sexos. Y que esto es así porque el "género" va más allá de las diferencias biológicas, al ser el resultado de interacciones sociales en las cuales las creencias y la estructura social, que define las relaciones de poder, juegan un papel fundamental.

Eagly y Carli (2003), por el contrario, concluyen que ciertas cualidades que responden al estereotipo "femenino", más fáciles de encontrar en las mujeres que en los hombres, favorecen un estilo de liderazgo contemporáneo, que resulta efectivo para hacer frente a la situación actual de continuos cambios y de intensa competencia. Así, desde este punto de vista, la mujer directiva presentaría determinadas ventajas en el liderazgo, aunque a la vez soportaría las desventajas derivadas de los prejuicios existentes a la hora de evaluar su competencia como directiva, especialmente en contextos empresariales masculinos (Rosen y Jerdee, 1973).

Cabe subrayar que, a pesar de las discrepancias encontradas en los resultados de las investigaciones sobre posibles influencias del género en los estilos de dirección, en ellas se ponía en evidencia la situación de desventaja en que están las mujeres en la empresa con respecto a los hombres por las barreras que encuentran para acceder a puestos directivos (Lyness y Thompson, 2000), o por la tendencia a promover a las mujeres a puestos directivos precarios con mayor riesgo de fracaso (Aslan y Ryan, 2008).

REVESCO No 105 - MONOGRÁFICO: La economía social y la igualdad de género - 
Problemas de género en la Cooperativa de trabajo asociado

En la Cooperativa de trabajo asociado (CTA), cuyo contexto organizativo se caracteriza por ser participativo e igualitario, también se manifiestan las barreras por género en el acceso a los puestos directivos. Las investigaciones de Berenguer et al (1999 y 2004), Fregidou (2004), Rivas y Sajardo (2004 y 2005), revelan el predominio del sexo masculino en los puestos directivos. Lo mismo ocurre con la composición de los consejos rectores (COCETA, 2004; Esteban, Gargallo y Pérez, 2010).

Esta situación de desigual presencia de las mujeres en funciones directivas en la CTA, y la falta de acuerdo en las investigaciones en cuanto a las posibles influencias de género en los estilos de dirección, lleva a plantearse hasta qué punto la CTA infrautiliza un recurso trascendental y escaso, como son las capacidades directivas, al no incorporar a tareas de alta dirección a las mujeres cooperativistas.

Como respuesta a dicha interrogante, y teniendo en cuenta que el contexto organizativo de la CTA, condicionará la actuación directiva, este trabajo indaga sobre tres ámbitos de la dirección de CTAs: a) Estilos de dirección que ejercen los directivos; b) Grado de ajuste de dichos estilos al contexto organizativo de la CTA y a los requerimientos del entorno competitivo; y c) Posible influencia del género en los estilos de dirección.

El trabajo ha sido estructurado en cuatro apartados: el primero tiene carácter introductorio; el segundo destaca las tendencias actuales en la Dirección de empresas; el tercero estudia los atributos institucionales de la CTA que tienen mayor impacto en el comportamiento directivo, y las capacidades directivas requeridas en el contexto actual de continuos cambios e intensa competencia; y, finalmente, el cuarto apartado, de carácter empírico, describe la metodología utilizada en la investigación y los resultados obtenidos.

\section{TENDENCIAS ACTUALES EN LA DIRECCIÓN DE EMPRESAS}

La Ciencia Administrativa proporciona varios enfoques sobre las actividades que deben realizar los directivos. El más difundido ha sido el enfoque clásico basado en las 
funciones administrativas de Fayol (1930), al que le han seguido enfoques basados en las características del directivo como decisor y como líder. Tal ha sido el caso de los trabajos de Simon (1947), March y Simon (1958), McGregor (1960), Likert (1961), Blake y Mouton (1964), Cyert y March (1965), Tannenbaum y Schmidt (1973), Mintzberg (1973) y Fiedler (1974).

Un importante avance en la forma de entender la dirección y el liderazgo en la empresa, que responde en gran medida a cómo se entiende el liderazgo en la actualidad, fue la aportación de Kotter (1988 y 1990). El liderazgo que concibe este autor se caracteriza por su flexibilidad, y su capacidad para crear redes de apoyo internas y externas, y por saber competir y colaborar. Para Kotter, los directivos de éxito consiguen sus objetivos mediante la participación de un grupo grande y variado de personas sobre las que ejercen escasa supervisión.

En línea con las aportaciones de Kotter, y ante la creciente evolución del entorno empresarial hacia situaciones de cambio abierto, las opciones que manejan los actuales sistemas de dirección, se orientan a generar en la empresa condiciones que posibiliten la creatividad y la innovación de manera continuada, así como la respuesta flexible y rápida a los cambios (KIiskberg, 1991). En esta línea, la empresa deberá desarrollar sistemas de vigilancia estratégica, sistemas de información fluidos y sistemas organizativos flexibles; teniendo en cuenta la definición amplia de las tareas, la superposición de funciones y un reparto más equitativo del poder (Galbraith, Lawler y Asociados, 1993).

En lo referido a los recursos humanos, se deberá promover la participación, la transparencia organizativa y la difusión sistemática de la información relevante sobre la situación de la empresa. Asimismo, deberán someterse a una continua revisión la política de remuneraciones y la formación del personal (García-Tenorio y Sabater, 2000). El modelo tradicional de imposición deberá reemplazarse por el de negociación y de cooperación.

Como Bacharach y Lawner (1980) proponen, en las organizaciones la negociación deberá ser la forma habitual de dirigir y, consecuentemente, el directivo deberá desarrollar las capacidades para ello.

REVESCO No 105 - MONOGRÁFICO: La economía social y la igualdad de género -

ISSN: 1885-8031 - www.ucm.es/info/revesco 
Este nuevo marco contempla como elementos claves a desarrollar en la empresa la descentralización de las decisiones, la implicación del trabajador y su formación, el enriquecimiento del trabajo, la ampliación de las tareas y la rotación de puestos, la visión compartida y, finalmente, el trabajo en equipo (Galbraith, Lawler y Asociados, 1993).

\section{LA FUNCIÓN DIRECTIVA EN LA COOPERATIVA DE TRABAJO ASOCIADO. FACTORES CONDICIONANTES}

Como es sabido, la CTA presenta una diferencia fundamental con la empresa capitalista tradicional: la singular configuración del sistema decisor de la empresa como consecuencia de la igualdad de derechos sobre el residual de la empresa de los socios trabajadores ${ }^{1}$.

En la empresa capitalista tradicional, tanto las decisiones para impulsar las necesarias adaptaciones de la empresa a contingencias internas o externas, es decir las decisiones de management, como las correspondientes a la afiliación de los miembros a la empresa, están centralizadas en el gerente, que actúa por delegación de los propietarios-accionistas (Alchian y Demsetz, 1972; Fama y Jensen ,1983a) y b)). Por el contrario, en la CTA únicamente las decisiones de management están asignadas a la gerencia, recayendo en los socios-trabajadores o en sus órganos representativos, las decisiones relacionadas con la incorporación o bajas de socios. Y en lo referido a las decisiones de management, los gerentes ven también muy limitada su actuación con relación a los gerentes de la empresa capitalista tradicional. ${ }^{2}$

\footnotetext{
${ }^{1}$ La forma institucional, tal como la estamos utilizando en este trabajo, se refiere al modo en que se asignan los derechos de propiedad y los derechos sobre el residual, y sus repercusiones sobre el comportamiento y la eficiencia productiva. La forma organizativa, en cambio, se refiere al conjunto de formas de dirección, normas y reglas, que implementan la forma institucional.

${ }^{2}$ En la CTA, la fase de control de las decisiones, es decir las fases de selección de alternativas y de control de la ejecución de las alternativas seleccionadas, recae en los propietarios que a la vez son miembros trabajadores de la empresa, y están representados en la Asamblea o en el Consejo. Esta situación les otorga atribuciones para rechazar aquellas decisiones relativas a adaptaciones organizativas que proponga la gerencia, que amenacen su posición de poder como trabajadores y socios en la empresa. Y en su calidad de trabajadores, deja abierta la posibilidad a que se produzcan comportamientos oportunistas en el cumplimiento de aquellas decisiones que habiendo sido seleccionadas por la Asamblea o el Consejo de la CTA, no convengan a sus intereses personales.
}

REVESCO Nº 105 - MONOGRÁFICO: La economía social y la igualdad de género -

ISSN: 1885-8031 - www.ucm.es/info/revesco 
Además, y como se verá a continuación, la igualdad de derechos sobre la empresa tendrá importantes efectos en el comportamiento de los socios, y en la forma en que se gobierna el trabajo en la CTA, todo lo cual va a condicionar la labor de los directivos.

\section{a) Comportamiento de los socios-trabajadores en la CTA.}

Desde una perspectiva motivacional-racional, el comportamiento individual en toda organización está determinado por lo siguientes factores: 1) Racionalidad limitada; 2) Disposición a cooperar; 3) Ámbito de actuación; 4) Alicientes o incentivos que ofrece la empresa (valores de conservación y recompensas materiales o inmateriales.); y 5) Racionalidad orgánica.

En base a ello, cabe considerar diferentes orientaciones del comportamiento en la empresa, según las personas dispongan de un menor o mayor ámbito de actuación, o área de ejercicio de racionalidad, y según su comportamiento esté guiado por incentivos del tipo recompensas materiales e inmateriales, o lo esté por incentivos del tipo valores de conservación; sin que estas preferencias tengan una relación directa con las tareas que realicen en la empresa, o con la posición jerárquica que ocupen ${ }^{3}$ (figura 3.1).

De acuerdo con este planteamiento, en la CTA, adquieren especial trascendencia las situaciones en que los incentivos predominantes corresponden a valores de conservación; es decir, situaciones en las que los incentivos están relacionados con objetivos de largo alcance, como son la dimensión, la supervivencia y el crecimiento de la empresa. Por tanto, y como los incentivos de esta naturaleza son la base para una lealtad organizativa, en la medida en que los incentivos para los socios trabajadores sean del tipo valores de conservación, estarán en mayor disposición para aprobar los cambios que desde los órganos de gobierno de la empresa se propongan.

El comportamiento de las personas se ve, también, afectado por otra forma de racionalidad que surge de la propia organización y que acaba por constituirse en una forma de conocimiento que Oliver Williamson (1985) denomina racionalidad orgánica. Todo ello

\footnotetext{
${ }^{3}$ Una justificación más detallada de este modelo y su aplicación a la CTA puede verse en Orellana et al (2003) y en Orellana y Monreal (2007).
}

REVESCO No 105 - MONOGRÁFICO: La economía social y la igualdad de género -

ISSN: 1885-8031 - www.ucm.es/info/revesco 
cristaliza en lo que Nelson y Winter (1982) denominan rutinas, y está en la base de un saber hacer que surge y emerge de la práctica organizativa, alimentando y formando, de manera emergente, la racionalidad de las personas.

El factor racionalidad orgánica es la base sobre la que se asienta el comportamiento de las personas, porque se desarrolla en la empresa a lo largo de su historia, y afecta a todos los miembros, teniendo un carácter más permanente, puede llevar a ampliar el área de racionalidad de los individuos por encima del nivel que la organización establece para ellos en el desempeño de sus tareas. Y el surgimiento y desarrollo de esta clase de racionalidad puede verse potenciado en el contexto organizativo de la $\mathrm{CTA}^{4}$.

Figura 3.1 Comportamiento en la empresa ${ }^{5}$

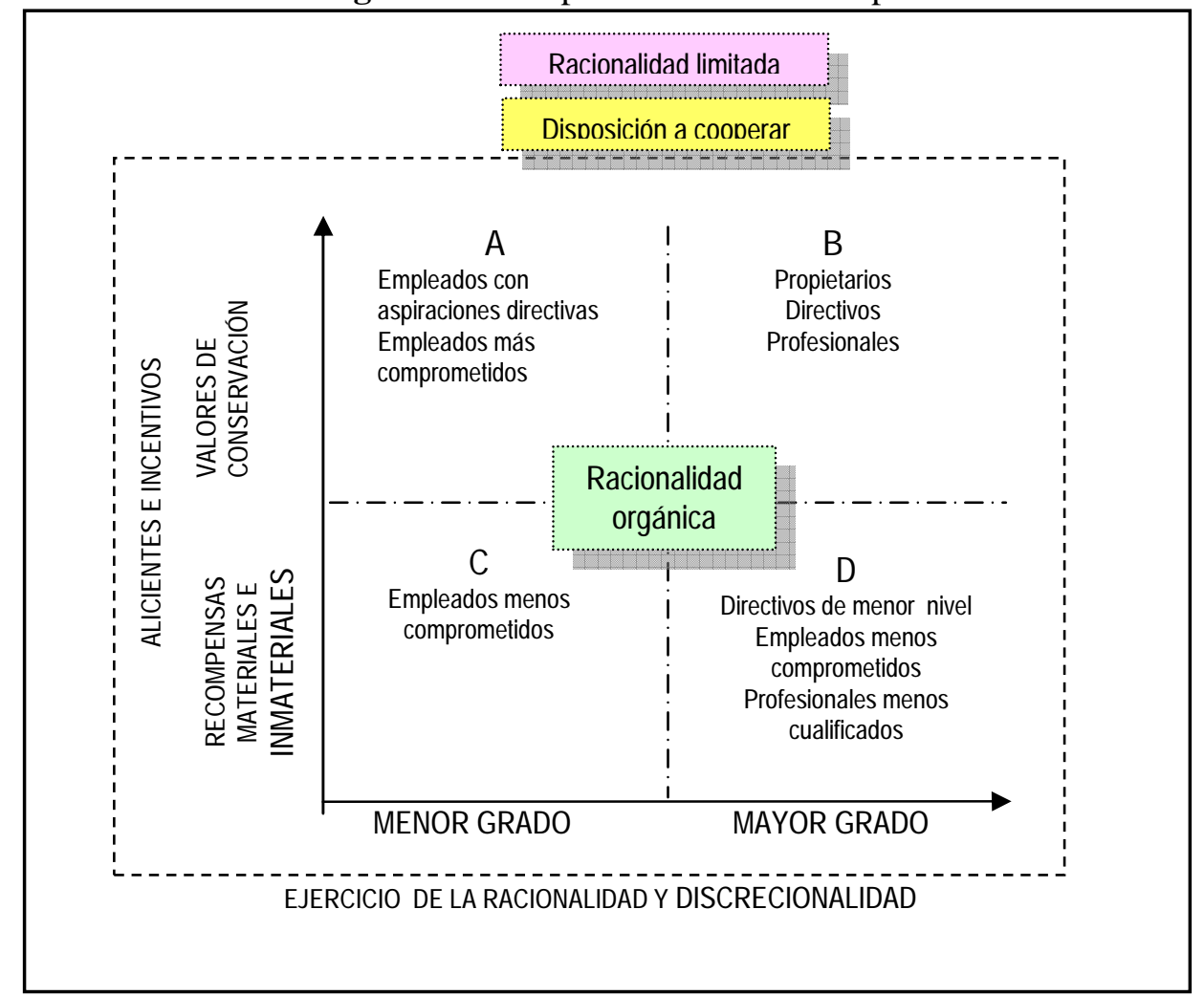

Basado en Barnard (1938); Simon (1947); March y Simon (1958); y Williamson (1985).

\footnotetext{
${ }^{4}$ En este sentido Hamilton (2009), observó que en organizaciones jerárquicas, las mujeres llevaban a cabo una actividad informal basada en la cooperación y en la ayuda mutua que no formaba parte de las tareas que tenían asignadas. Dicha actividad consistía en formas de coordinación horizontal que ellas mismas definían como "proteger y compartir" y que beneficiaba a la empresa pero que al no ser visible, no era remunerada.

${ }^{5}$ El modelo presentado (figura 3.1) resume diferentes formas de orientación del comportamiento. Las áreas A, B, C y D, encuadradas dentro del contexto empresarial, reflejan posibles orientaciones del comportamiento de los distintos miembros. En la intersección de las paralelas a los ejes de coordenadas se ha situado el factor racionalidad orgánica, para indicar que ésta afecta a todos los miembros de la organización, ya sean empleados, profesionales o directivos, mientras que racionalidad limitada y disposición a cooperar se representan como factores exógenos al contexto empresarial, y no pueden modificarse con actuaciones de diseño organizativo.
}

REVESCO N 105 - MONOGRÁFICO: La economía social y la igualdad de género -

ISSN: 1885-8031 - www.ucm.es/info/revesco 


\section{b) Gobierno del trabajo en la CTA}

Uno de los elementos distintivos de la CTA es la particular forma de gobernar las relaciones laborales, debido a la doble condición de trabajador y socio de los miembros.

Desde la Teoría de los Costes de Transacción, las relaciones laborales pueden plantearse como transacciones cuya naturaleza va a depender de la especificidad del trabajador y de la separabilidad de las tareas que realiza. La especificidad del trabajo vendrá determinada por el grado en que, para la empresa, el trabajador es idiosincrásico, y la separabilidad dependerá del grado de interrelación de las tareas que realiza el trabajador con otros puestos de trabajo. Desde esta perspectiva, el gobierno del trabajo consistirá en diseñar contratos e incentivos eficientes para cada una de las formas (Williamson, 1985).

Si se representa la especificidad de los activos humanos con $K_{l}$ cuando el trabajador es idiosincrásico $y$ con $K_{0}$ cuando no lo es; y con $S_{l}$ cuando se trata de trabajo interrelacionado y con $S_{0}$ la situación contraria, podrán distinguirse cuatro formas de situaciones laborales que la dirección de la empresa deberá gestionar: $K_{0} S_{0} ; K_{0}, S_{1} ; K_{1}, S_{0} ;$ y $K_{1}, S_{1}$.

De acuerdo con lo anterior, en la CTA la condición de socios de sus trabajadores les da carácter de trabajador idiosincrásico, con lo cual las formas de gobierno del trabajo predominantes se reducen a $K_{1}, S_{0} ;$ y $K_{1}, S_{1}{ }^{6}$. Esto es así porque cuando el trabajador es idiosincrásico $\left(K_{l}\right)$ la empresa otorgará una considerable estabilidad en el empleo, intentará “socializar” a los miembros y fomentará la lealtad y una actitud abierta al cambio.

\section{Efecto en los estilos directivos}

Las relaciones contractuales propias de la CTA, el comportamiento de los socios trabajadores, y el gobierno de las relaciones laborales, condicionarán la función directiva. La gerencia verá restringida su actuación por el poder negociador de los socios-trabajadores, que le otorgan los derechos sobre el residual de la CTA, y su participación directa o indirecta en la

\footnotetext{
${ }^{6}$ Cuando el trabajador no es idiosincrásico $\left(K_{0}\right)$, la relación contractual es débil ya que habitualmente se trata de trabajadores fácilmente sustituibles, por ello la empresa suele subcontratarlo cuando el trabajo que realiza no está interrelacionado con otras tareas, o realizarlo mediante contratación temporal si está interrelacionado.
}

REVESCO No 105 - MONOGRÁFICO: La economía social y la igualdad de género -

ISSN: 1885-8031 - www.ucm.es/info/revesco 
dirección de la empresa. Con lo cual las acciones que la gerencia pretenda llevar a cabo, habrán de ser necesariamente negociadas con los implicados.

Asimismo, los procesos de comunicación y de liderazgo propios de la CTA, unido a la participación de los socios en la dirección de la empresa a través de sus órganos rectores, impulsarán el desarrollo de la racionalidad orgánica, y dotarán a los socios-trabajadores de una amplia área de ejercicio de la racionalidad. Con lo cual el comportamiento de los socios trabajadores, responderá preferentemente a la modalidad B del modelo presentado en la figura 3.1. Esto requerirá de la gerencia capacidades especiales para actuar en un contexto en que los miembros disponen de información tanto del trabajo que realizan sus iguales como de las iniciativas y políticas que adopten sus directivos, así como de amplias atribuciones para realizar sus tareas.

En lo referente al factor "gobierno del trabajo", la gerencia deberá potenciar el trabajo en equipo, capitalizar las capacidades de los trabajadores idiosincrásicos y diseñar incentivos colectivos, puesto que en la CTA la relación laboral $K_{l} S_{1}$ es la predominante, siendo los trabajadores portadores de capacidades y habilidades distintivas, fundamentales para la empresa. Esta clase de relación laboral debe gobernarse mediante soluciones organizativas negociadas, sistemas de comunicación fluidos, equipos de trabajo e incentivos colectivos. Teniendo en cuenta que la relación laboral descrita está próxima a la que se da en organizaciones tipo clan en las que "...se reducen las diferencias entre los objetivos de los individuos y los de la organización y se crea un poderoso sentido de comunidad" (Ouchi, 1980: 136).

\section{GÉNERO Y CAPACIDADES DIRECTIVAS EN LA CTA}

Hasta aquí ya han sido especificadas las capacidades directivas requeridas para actuar en entornos complejos y de intensa competencia, y también la forma en que condicionarán el trabajo directivo factores que son consecuencia de las propiedades institucionales de la CTA.

Corresponde ahora investigar qué perfiles directivos existen en la CTA, el grado de correspondencia de dichos perfiles con las capacidades directivas necesarias para actuar en 
entornos competitivos como los actuales, y las posibles influencias de género en la configuración de dichos perfiles.

Con ese fin, se planteó la realización de entrevistas a directivos y directivas de nivel alto, incluidos gerentes.

Las entrevistas debían proporcionar información fundamental sobre la forma como entendían los directivos su trabajo, las tareas que realizaban habitualmente, y cómo se relacionaban con las personas con las cuales se vinculaban en la empresa, incluidos los subordinados.

Las entrevistas proporcionaron información sobre 340 variables. Después de un proceso de depuración de la información, el número de variables se redujo a 256. Una vez codificada la información, se procedió a la realización de un análisis cluster en dos etapas y mediante los correspondientes análisis de homogeneidad se obtuvieron dos perfiles directivos en la CTA.

\section{Metodología}

La información empírica básica se obtuvo de un cuestionario que se utilizó como guía para el entrevistador. En dicho cuestionario se incluyeron preguntas relacionadas con el trabajo que realizaban directivos (hombres y mujeres) de nivel medio y alto de CTAs, orientadas a conocer la forma en que los directivos entendían debía llevarse a cabo la dirección de la empresa. Esto permitiría al investigador obtener la información necesaria para determinar estilos y capacidades directivas existentes en la CTA, y estudiar influencias de género en dichas capacidades.

La muestra aleatoria la conformaron 44 CTAs que fueron seleccionadas de un universo de 156 empresas, situadas en la Comunidad Valenciana, que contaban con un número de trabajadores socios y no socios superior a 25. Las empresas estaban incluidas en la Base de datos SABI (Sistema de Balances Ibéricos) y previa carta de solicitud de entrevista pudieron 
ser entrevistados 19 mujeres y 25 hombres que ocupaban en la empresa puestos directivos altos.

La mayoría de las empresas entrevistadas contaban con una plantilla superior a 25 trabajadores y menor a 60 , considerando trabajadores socios y no socios. En promedio, el número de trabajadores socios era del $78 \%$, aunque cabe subrayar que la proporción de socios no trabajadores era mayor en las empresas de mayor tamaño.

La antigüedad de las empresas entrevistadas estaba comprendida entre los 15 y 24 años.

\section{Obtención de la información}

Dado que el objetivo último de la investigación era conocer el contenido de las tareas que llevan a cabo los directivos (hombres y mujeres) de CTA y la percepción que tenían acerca de la naturaleza de su trabajo, se optó por utilizar una variante del Método de la observación estructurada, metodología utilizada en investigaciones similares a ésta, y divulgada en la investigación pionera realizada por Henry Mintzberg sobre la naturaleza del trabajo de los directivos (Mintzberg, 1973).

El método consiste básicamente en la realización de entrevistas a directivos, tomando como referencia un cuestionario elaborado por el investigador, que luego éste interpreta y tabula de acuerdo a los objetivos planteados en la investigación.

\section{Tratamiento estadístico de las entrevistas}

La información que se obtuvo de cada directivo recogida en cada cuestionario se tradujo en 256 variables que fueron codificadas y archivadas en una matriz de 44 filas y 256 columnas. Esta matriz conformó una base de datos con información para ser procesada en el programa estadístico SPSS.

Dado que cada directivo quedaba identificado de acuerdo a los atributos recogidos en 256 variables, se procedió a agrupar las posibles respuestas a las 256 variables, en conjuntos de variables, de acuerdo al contenido de las mismas, con base a criterios de analogía y 
complementariedad. Así, las distintas opciones de respuesta de los directivos a cada variable quedaban representadas por dos estados (si o no), y cada uno de los conjuntos en que habían sido agrupadas, proporcionaba los distintos valores que podían tomar cada variable, y por tanto una característica del directivo o directiva en cuestión ${ }^{7}$.

Se pretendía conseguir una metodología capaz de formar grupos de directivos que internamente albergasen a directivos que hubiesen dado una respuesta similar a las cuestiones planteadas.

De esta forma, la pertenencia de un directivo a un determinado grupo indicaría que posee unas características exclusivas que lo hacen distinto a otros directivos del resto de grupos. Y, dichas características servirán de base para identificar ("etiquetar") al directivo según éste posea o no determinadas características. Así cada directivo podrá ser identificado con $\mathrm{m}$ etiquetas, siendo $\mathrm{m}$ la respuesta específica que ha dado a la pregunta contenida en el conjunto.

Con la información proporcionada por los directivos en las entrevistas, se configuraron 47 conjuntos de preguntas, que en algunos casos eran de carácter unitario, como el origen de la CTA, o sexo del directivo. Lo que originó un tratamiento especial de estos casos.

Así, en el caso de conjuntos compuestos por una única variable, el análisis consistió en la simple clasificación o categorización de los directivos en base a los valores de las variables, estableciendo un número de categorías próximo a cuatro, en vías de unificar las características de todas las variables resultantes. Uno de los conjuntos de preguntas ilustra esta situación: "Estudios realizados", compuesto por una única variable, posibilitaba tres respuestas: "elementales o medios", "diplomado", y "licenciado", son estas posibles respuestas las que establecen las tres categorías y clasifican claramente a los directivos.

\footnotetext{
${ }^{7}$ Dada la distinta forma en que se medían las variables (información medida en intervalos, información que corresponde a atributos, información expresada en escala nominal, etcétera.), los métodos estadísticos de reducción de datos como: el análisis de componentes principales, el análisis factorial, y el análisis de correspondencias, no pudieron ser utilizados
} 
En el caso de conjuntos compuestos por más de una variable se procedió de la forma que se describe a continuación.

a) Cluster primer análisis

Para cada conjunto se hizo un análisis cluster para agrupar a los directivos de acuerdo a las variables que forman el conjunto, estableciéndose el número de clusters ${ }^{8}$ en 3,4 , y 5 (el análisis cluster se realizó por el método de $\operatorname{Ward}^{9}$ ). A continuación, en cada conjunto se realizó un análisis de homogeneidad (Chi-cuadrado de Pearson) del que se obtuvieron cluster de "cualidades directivas" en los conjuntos que se describen en la tabla 4.1.En el resto de conjuntos, las pruebas de homogeneidad no detectaron diferencias significativas en las cualidades directivas.

Tabla 4.1 Conjuntos de preguntas contenidas en el cuestionario que proporcionaron diferencias entre directivos

\begin{tabular}{l|l|l}
\hline & \multicolumn{1}{|c|}{ Conjuntos } & \multicolumn{1}{|c}{ Objetivo de las preguntas } \\
\hline 1 & Origen de la Cooperativa & $\begin{array}{l}\text { Identificar si la cooperativa había sido creada } \\
\text { como tal desde sus inicios o provenía de la } \\
\text { transformación de una empresa capitalista } \\
\text { tradicional en crisis }\end{array}$ \\
\hline 2 & Sexo del directivo & $\begin{array}{l}\text { La condición de género se utilizó como una } \\
\text { característica más de los directivos }\end{array}$ \\
\hline 3 & Estudios de los directivos & Identificar la formación de los directivos \\
\hline 4 & $\begin{array}{l}\text { Opinión del directivo sobre el } \\
\text { puesto que ocupa }\end{array}$ & $\begin{array}{l}\text { Identificar conocimientos y cualidades personales } \\
\text { que considera necesarias para el desempeño de su } \\
\text { puesto y sus expectativas en el puesto }\end{array}$ \\
\hline 5 & $\begin{array}{l}\text { Tareas prioritarias a realizar por el } \\
\text { directivo }\end{array}$ & $\begin{array}{l}\text { Identificar la visión del directivo respecto de su } \\
\text { propio puesto }\end{array}$ \\
\hline 6 & $\begin{array}{l}\text { Conocimientos que necesita el } \\
\text { directivo para su trabajo }\end{array}$ & $\begin{array}{l}\text { Identificar los temas que el directivo considera más } \\
\text { importantes }\end{array}$ \\
\hline 7 & $\begin{array}{l}\text { Aspectos en los que el directivo se } \\
\text { considera deficitario }\end{array}$ & $\begin{array}{l}\text { Identificar prioridades que da el directivo a } \\
\text { determinados ámbitos de actuación }\end{array}$ \\
\hline 8 & $\begin{array}{l}\text { Distribución del tiempo del } \\
\text { directivo }\end{array}$ & $\begin{array}{l}\text { Identificar las tareas que más tiempo ocupan al } \\
\text { directivo, discriminándolas según su trascendencia } \\
\text { para la empresa. }\end{array}$ \\
\hline 9 & $\begin{array}{l}\text { Opinión sobre aspectos críticos de } \\
\text { su empresa (favorables y } \\
\text { desfavorables) }\end{array}$ & $\begin{array}{l}\text { Identificar capacidades directivas de dirección de } \\
\text { la empresa }\end{array}$ \\
\hline 10 & $\begin{array}{l}\text { Conocimiento del sector } \\
11\end{array}$ & $\begin{array}{l}\text { Opinión sobre el futuro del sector } \\
\text { Identificar capacidades directivas }\end{array}$ \\
\hline & Opinión sobre la formación de los & Identificar capacidades directivas \\
\hline
\end{tabular}

\footnotetext{
${ }^{8}$ Dado que el número de directivos es 44 una agrupación en 3 de aproximadamente 14 sería la ideal, una de 4 en aproximadamente 11 asimismo lo sería, y, también, una de 5 con 8 directivos cada una.

${ }^{9}$ Se utilizó el método de Ward dado que, como es sabido, es el más exigente en la determinación de los niveles de agrupación, e interesaba crear las máximas discrepancias entre los grupos que se pretendían formar
}

REVESCO N 105 - MONOGRÁFICO: La economía social y la igualdad de género -

ISSN: 1885-8031 - www.ucm.es/info/revesco 


\begin{tabular}{c|l|l}
\hline & RRHH & recursos humanos \\
\hline 13 & $\begin{array}{l}\text { Estilo de liderazgo que ejerce el } \\
\text { directivo }\end{array}$ & Detectar estilos predominantes de dirección \\
\hline 14 & $\begin{array}{l}\text { Actitud del directivo hacia los } \\
\text { subordinados }\end{array}$ & $\begin{array}{l}\text { Identificar la forma en que el directivo delega } \\
\text { autoridad }\end{array}$ \\
\hline 15 & Directivo y participación & $\begin{array}{l}\text { Identificar mecanismos de participación en la } \\
\text { empresa y actitud del directivo frente a la } \\
\text { participación }\end{array}$ \\
\hline 16 & Utilización del tiempo & $\begin{array}{l}\text { Mediante un Test se contrastan las actividades } \\
\text { desarrolladas por el directivo }\end{array}$ \\
\hline 17 & Delegación de autoridad & $\begin{array}{l}\text { Mediante un Test se contrasta la actitud del } \\
\text { directivo hacia la participación }\end{array}$ \\
\hline 18 & Asertividad del directivo & $\begin{array}{l}\text { Mediante un Test se detecta la actitud general del } \\
\text { directivo en su relación con los subordinados }\end{array}$ \\
\hline
\end{tabular}

b) Cluster segundo análisis

Tras el primer análisis estadístico, y dado que la información quedó reducida a $\mathrm{M}$ variables categóricas pertinentemente etiquetadas, se procedió a un segundo análisis cluster y, como resultado de este análisis, cada directivo quedó asignado a un único grupo, al que reúne directivos con algunas de las características coincidentes con las propias y que están contenidas en las $\mathrm{M}$ variables categóricas señaladas antes.

A continuación, y al igual que en el primer análisis cluster, se realizó un análisis de homogeneidad, para contrastar la existencia de comportamiento homogéneo de cada grupo creado con relación a cada una de las variables.

De esta forma, se consiguió que cada directivo quedase identificado de manera unívoca sobre la base de toda la información que se disponía sobre él.

\section{Resultados de la investigación}

Después del análisis cluster final, se verificó la existencia de dos perfiles directivos determinados por los siguientes conjuntos:

a) Origen de la cooperativa (conjunto 1). Se distinguen dos grupos de sociedades según la empresa sea desde su origen una CTA, o su origen esté en la transformación de una empresa en crisis.

b) Sexo del directivo (conjunto 2)

c) Estudios realizados (Conjunto 3). Los grupos de directivos están determinados por aquellos REVESCO No 105 - MONOGRÁFICO: La economía social y la igualdad de género -

ISSN: 1885-8031 - www.ucm.es/info/revesco 
que tienen estudios universitarios y quienes tienen otros tipos de estudios.

d) Capacidades personales (Conjuntos 13, 14, 15, y 17). Un grupo de directivos presenta una clara orientación a ejercer liderazgo como cualidad para la dirección de la CTA. Los restantes directivos se orientan más a una dirección participativa basada en la comunicación fluida y en la solución negociada de los problemas.

e) Conocimientos necesarios (Conjunto 6, 9, 11 y 12). Se presentan dos tendencias: una correspondiente a aquellos directivos que incluyen a los recursos humanos entre los conocimientos fundamentales para la dirección de empresas; y otra correspondiente a directivos que dan más importancia a conocimientos técnicos (finanzas, administración, etcétera).

f) Tareas primordiales (conjunto 5 y 16). Se distinguen dos grupos de directivos: aquellos que entienden su tarea directiva básicamente como: coordinar actividades de todo tipo; y otros directivos que entienden su tarea más relacionada con el control, y con aspectos técnicos.

En resumen, de acuerdo a las respuestas de las entrevistas, y en base a los atributos: origen de la sociedad, sexo del directivo, estudios realizados, tareas primordiales, conocimientos necesarios, y capacidades personales, en la CTA valenciana se configuran dos perfiles de directivos, según se representan en la tabla 4.2

Tabla 4.2 Perfiles directivos en las CTA

\begin{tabular}{|c|c|c|}
\hline ATRIBUTOS & PERFIL A & PERFIL B \\
\hline Directivo & Mujer & Hombre \\
\hline Origen de la CTA & CTA desde un comienzo & $\begin{array}{l}\text { Transformación empresa } \\
\text { capitalista }\end{array}$ \\
\hline Estudios & No universitarios & Universitarios \\
\hline $\begin{array}{l}\text { Tareas primordiales que realizan } \\
\text { los directivos entrevistados }\end{array}$ & Coordinar actividades & $\begin{array}{l}\text { Aplicación de } \\
\text { conocimientos técnicos }\end{array}$ \\
\hline $\begin{array}{l}\text { Conocimientos que el directivo } \\
\text { considera necesarios para } \\
\text { ejercer el puesto directivo. }\end{array}$ & $\begin{array}{l}\text { Preferentemente gestión de } \\
\text { recursos humanos }\end{array}$ & $\begin{array}{l}\text { Conocimientos técnicos de } \\
\text { gestión }\end{array}$ \\
\hline $\begin{array}{l}\text { Capacidades personales que el } \\
\text { directivo señala como necesarias } \\
\text { para desempeñar el puesto } \\
\text { directivo }\end{array}$ & Capacidad de negociación & $\begin{array}{l}\text { Dirigir personas } \\
\text { ejerciendo liderazgo }\end{array}$ \\
\hline
\end{tabular}

REVESCO No 105 - MONOGRÁFICO: La economía social y la igualdad de género -

ISSN: 1885-8031 - www.ucm.es/info/revesco 
El directivo de perfil A, no ha cursado estudios universitarios, aunque sí ha hecho cursos de perfeccionamiento en dirección de empresas, y entiende la gerencia de una manera cualitativa en que la capacidad de comunicación y de negociación son fundamentales.

El directivo de perfil $\mathrm{B}$, tiene cursados estudios universitarios, piensa que la dirección de la empresa es una cuestión compleja que requiere conocimientos técnicos, y entiende la gerencia en términos de liderazgo en la línea del tipo autoritario-benévolo. Este grupo de directivos en su mayoría proviene de empresas capitalistas tradicionales que al entrar en crisis adoptaron la forma CTA, al contrario que las empresas de los directivos de Perfil A, que adoptaron la forma CTA al momento de su creación.

\section{CONCLUSIONES}

La investigación no arroja resultados categóricos acerca de perfiles directivos existentes en la CTA, pero es posible distinguir determinados atributos directivos que derivan en dos formas distintas de entender el liderazgo (por simplificación se denominarán como perfiles A y B a cada una de las formas).

En primer lugar, cabe destacar las tecnologías de tipo cualitativo que se dan en el perfil A y las de tipo cuantitativo que se dan en el perfil B.

El directivo de perfil A, tiene orientado su comportamiento hacia la coordinación y a la negociación, y concede especial importancia a los recursos humanos.

Estos atributos suponen una mayor correspondencia con las capacidades directivas que se requieren para competir con éxito en la era actual y para actuar en contextos organizativos participativos como el de la CTA. De la misma forma, dichos atributos facilitarán, sin duda, las relaciones laborales con trabajadores idiosincrásicos como lo son sus socios trabajadores.

Con respecto al perfil directivo $\mathrm{B}$, este responde en mayor medida a un liderazgo clásico que se sustenta en la autoridad que otorga el ser directivo y, por tanto, más ajustado a 
las relaciones contractuales típicas de empresa capitalista que a las de una empresa participativa e igualitaria como la CTA.

Los atributos del directivo de perfil B no tienen buen encaje con los requerimientos de la dirección contemporánea, ni con los requerimientos de un contexto participativo como lo es el de la CTA.

Respecto de las cualidades directivas necesarias en la era actual, el directivo de perfil A presenta una mayor correspondencia en algunos aspectos (capacidad de negociación, información, etcétera) que el directivo de perfil B, y éste presenta algunas ventajas derivadas de su capacidad técnica.

La cuestión aquí, es si cabe emitir un juicio en defensa de una u otra forma de entender el trabajo directivo.

En cualquier caso, y de acuerdo al análisis realizado en los primeros epígrafes; para la CTA, resulta evidente que el perfil A tiene mayor correspondencia con el contexto organizativo-contractual de la empresa, lo que facilitará su equilibrio interno.

Por tanto, en el contexto de la CTA valenciana, se confirman las tesis de Eagly y Carli (2003) en el sentido de que existe un estilo que responde al estereotipo de femenino que favorece un liderazgo contemporáneo, acorde con la situación actual.

Asimismo, y en línea con el resultado de la investigación de Berenguer et al (2004), realizada en CTAs valencianas, al igual que esta investigación, las características del liderazgo de estilo femenino orientado a la coordinación y a la negociación tienen un mejor encaje con la forma institucional cooperativa de trabajo asociado.

REVESCO N 105 - MONOGRÁFICO: La economía social y la igualdad de género -

ISSN: 1885-8031 - www.ucm.es/info/revesco 


\section{BIBLIOGRAFÍA}

ALCHIAN, A., y DEMSETZ, H. "Production, information cost, and economic organization". American Economic Review. 62, 1972 (december), 777-795. [Traducción al castellano: "Producción, Costes de Información y Organización Económica”, en Louis Putterman, ed., La naturaleza económica de la empresa. Alianza, Madrid, 1994].

ASLAN, S.A. y RYAN, M.K. "The road to the glass cliff: Differences in the perceived suitability of men and women for leadership positions in succeeding and failing organizations". Leadership Quarterly. Vol 19, № 5, 2008, pp. 530-546.

BACHARACH, S.B. y LAWLER, EDWARD J. Power and Politics in Organizations. San Francisco, Jossey Bass, 1980.

BARNARD, C. I. The functions of the executive. Harvard University Press. Cambridge, Mass, 1938. [Traducción al castellano: Las funciones de los elementos dirigentes. Instituto de Estudios Políticos, Madrid. 1959].

BERENGUER, G. CASTEllví, J.M. CERVER, E. JUAN, C. TORCAL, V.R. DE LA TORRE, A. El laberinto de cristal. Un estudio sobre el acceso de las mujeres a los puestos de dirección en las cooperativas de trabajo asociado valencianas. Dificultades y oportunidades. FEVECTA, Valencia, 1999.

BERENGUER, G.; CERVER, E. DE LA TORRE, A. TORCAL, R. "El estilo directivo de las mujeres y su influencia sobre la gestión del equipo de trabajo en las cooperativas valencianas". CIRIEC-España, no 50, 2004, pp. 123-149.

BLAKE, R. R., y MOUTON, J. S. The Managerial Grid, Gulf Publishing, Houston, 1964.

CYERT, R. y MARCH, J. Teoría de las decisiones económicas en la empresa. Herrero Hermanos Sucesores, S.A. México,1965.

COCETA. CONFEDERACIÓN ESPAÑOLA DE COOPERATIVAS DE TRABAJO ASOCIADO. Estudio sobre la realidad social y laboral de las mujeres en el Cooperativismo de trabajo asociado en España. 2004. http://www.coceta.com.

EAGLY, A. H.; y CARLI, L.L. “The female leadership advantage: An evaluation of the evidence”. Leadership Quarterly, vol. 14, nº 6, 2003, pp. 807-834.

ESTEBAN, M. L.; GARGAllO, A.; y PÉREZ, F. J. “Composición del Consejo rector y género en las Cooperativas turolenses". REVESCO, nº 101, 2010, pp. 7- 27.

REVESCO No 105 - MONOGRÁFICO: La economía social y la igualdad de género -

ISSN: 1885-8031 - www.ucm.es/info/revesco 
FAMA, E., y JENSEN, M. "Separation of Ownership and Control", Journal of Law and Economics, n² 26, 1983a pp.301-326.

FAMA, E., y JENSEN, M. “Agency Problems and Residual Claims”, Journal of Law and Economics, no 26, 1983b, pp.327-349.

FAYOL, H. Industrial and General Administration. Londres, 1930

FIEDLER, F. E. "The contingency model. New directions for leadership utilization"; [en KOONTZ, H., Y OTROS (eds.): Management, McGraw-Hill, 1980].

FREGIDOU, M.: “¿Es necesario equilibrar el poder en las cooperativas entre mujeres y hombres?". CIRIEC-España, nº 50, 2004, pp. 151-163.

GALBRAITH, J. R., LAWLER, E. E., Y ASOCIADOS. Organizing for the Future, The Jossey-Bass, San Francisco, 1993.

GARCÍA-TENORIO, J. y SABATER, R. (Coord.): Fundamentos de Dirección y Gestión de Recursos Humanos. Thomson. Madrid, 2000.

HAMILTON, J.L. "Caring/Sharing: Gender and Horizontal Co-ordination in the Workplace”. Gender, Work and Organization. 2009, pp.1-26.

KLIKSBERG B. "Las Perspectivas de la Gerencia Empresarial en los Años Noventa". Pensamiento Iberoamericano, $\mathrm{n}^{\circ} 19,1991$, pp. 141-163.

KOTTER, J. P. The Leadership Factor. Free Press, New Cork,1988. [Traducción al castellano: El Factor Liderazgo, Díaz de Santos, Madrid, 1990].

KOTTER, J. P. A Force for Change: How Leadership Differs from Management. Free Press, New York, 1990.

LIKERT, R. New Patterns of Management, McGrawHill, New York, 1961.

LYNESS, K.S. y THOMPSON, D.E. "Climbing the Corporate Ladder: Do Female and Male Executives Follow the Same Route?". Journal of Applied Psychology, Vol. 85, No. I, 2000, pp. 86-101.

MARCH, J.G., y SIMON, H.A. Organizations. John Wiley and Sons. New York, 1958. [Traducción al castellano: Teoría de la Organización, Ariel. Barcelona.1977].

MCGREGOR, D. The Human Side of Enterprise, McGraw Hill, New York, 1960.

MINTZBERG,H. The Nature of Managerial Work, Harper \& Row, New York, 1973.

[Traducción al castellano: La naturaleza del trabajo directivo, Ariel, Barcelona, 1991].

NELSON, R. and WINTER, S. An Evolutionary Theory Of Economic Change, The Belknap Press of Harvard University Press, Cambridge and London, 1982.

REVESCO N 105 - MONOGRÁFICO: La economía social y la igualdad de género -

ISSN: 1885-8031 - www.ucm.es/info/revesco 
ORELLANA, W.; CHAVES, R. RUEDA, C. Antecedentes institucionales y gobierno de la empresa de trabajo asociado. Efectos de la descentralización contractual en el proceso de toma de decisiones. En IX Jornadas de Investigadores en economía social y cooperativa. Universidad de Valladolid. Valladolid, 2003.

ORELlANA, W.; y MONREAL, M. Control, incentivos y comportamiento en las organizaciones. ACDE. Valencia-España, 2007.

OUCHI, W. G.: "Markets bureaucracies and clans", Administrative Sciencie Quarterly, vol. 25, 1980, pp. 124-141.

RIVAS, M.A. y SAJARDO, A. "La desigual participación de hombres y mujeres en la economía social: teorías explicativas”. CIRIEC-España, no 50, 2004, pp. 77-103.

RIVAS, M.A.. y SAJARDO, A. "La diferente participación laboral de las mujeres entre las cooperativas y las sociedades laborales”. CIRIEC-España, nº 52, 2004, pp. 267 - 278.

ROSEN, B.; y JERDEE, T.H. "The influence of sex-role stereotypes on evaluations of male and female supervisor behaviour". Journal of Applied Psychology, 57, 1973, 44-48.

SIMON, H. A. Administrative Behavior. A study of decision making processes in administrative organization. Harper \& Brothers. New York, 1947 [Traducción al castellano: El comportamiento administrativo. Estudio de los procesos de adopción de decisiones en la organización administrativa. Aguilar. Madrid. 1972].

STEWART, A.J. y McDERMOTT, C. "Gender in Psychology". Annual Review of Psychology, Vol. 55, 2004, pp. 519-544.

TANNENBAUN, R., y SCHMIDT, W. H. "How to choose a leadership pattern". Harvard Business Review, vol. 51, n 3, 1973, pp. 162-180.

VECCHIO, R.P. "In search of gender advantage", The Leadership Quarterly, n 14, 2003, pp. $835-850$.

WILLIAMSON, O.E. The Economic Institutions of Capitalism. The Free Press, New York, 1985. [Traducción al castellano: Las instituciones económicas del capitalismo. Fondo de Cultura Económica, México, 1989].

REVESCO No 105 - MONOGRÁFICO: La economía social y la igualdad de género -

ISSN: 1885-8031 - www.ucm.es/info/revesco 